\title{
Revisão da Literatura sobre a Eficácia da Intervenção Psicológica no Tratamento do Lúpus Eritematoso Sistêmico
}

\author{
Sílvia Fernanda Lima de Moura $\mathrm{Cal}^{1}$ \\ Fundação Baiana para o Desenvolvimento das Ciências
}

\begin{abstract}
RESUMO - Manifestações neuropsiquiátricas são comuns no lúpus eritematoso sistêmico (LES), especialmente depressão, ansiedade e psicose. O estresse psicológico e o uso de corticóide têm sido responsabilizados pelas manifestações psicopatológicas. Objetivou-se realizar revisão de literatura sobre a eficácia da intervenção psicológica no tratamento do LES, utilizandose pesquisas em bases de dados, através dos descritores "psychotherapy" and "lupus", incluindo-se os ensaios clínicos randomizados e os estudos prospectivos. Foram encontrados seis artigos, sendo quatro ensaios clínicos randomizados e dois estudos prospectivos. Cinco artigos encontraram evidências de acentuada melhora nos pacientes que tinham acompanhamento psicológico e apenas um não encontrou tal evidência. Concluiu-se que a intervenção psicológica pode ser uma ferramenta importante no tratamento do LES.
\end{abstract}

Palavras-chave: lúpus eritematoso sistêmico, psicoterapia, doença autoimune.

\section{Literature Review of Efficacy of Psychological Interventions in the Treatment of Systemic Lupus Erythematosus}

\begin{abstract}
Neuropsychiatric manifestations, especially depression, anxiety and psychosis, are common in systemic lupus erythmatosus (SLE). The psychological stress and the use of corticoids have been blamed for these psychopathological manifestations. A literature review was realized on the efficacy of psychological interventions in the treatment of SLE, using research data bases, through the descriptors "psychotherapy" and "lupus", including randomized clinical trials and prospective studies. Six articles were found, of these, four were randomized clinical trials, and two prospective studies. Five articles related evidence of pronounced improvement in patients that had psychological follow-up and one article did not found such evidence. Psychological intervention can be an important tool in the treatment of SLE.
\end{abstract}

Keywords: systemic lupus erythemathosus, psychotherapy, autoimmune disease.

O lúpus eritematoso sistêmico (LES) é uma doença crônica e autoimune capaz de atingir vários órgãos, inclusive o sistema nervoso. As manifestações neuropsiquiátricas são comuns no LES, variando amplamente de 5\% a 83\% (Ampelas, Wattiaux \& Van Amerongen, 2001), 17\% e 75\% (Miguel et al.,1994), e até 91\% segundo outros estudos (Abdel-Nasser, Ghaleb, Mahmoud, Khairy \& Mahmoud, 2008; Lessa, Santana, Lima, Almeida \& Santiago, 2006), e entre elas a depressão é a mais prevalente, mas também podem ocorrer psicose, convulsões, cefaléias, tonturas, meningite, estados delirantes e transtorno de ansiedade (Nery et al., 2007).

Alguns estudos têm associado essas manifestações clínicas a anticorpos, como o anti Pribossomal, anti-SSA, anti-DNA, anticardiolipina e antifosfolípides (Abdel-Nasser at al., 2008; Ayache \& Costa, 2005; Olsen et al., 1991), entretanto há trabalhos que mostram resultados divergentes (Eber, Chapman \& Shoenfeld, 2005; Nery et al., 2008).

O LES, devido a sua complexidade, afeta toda a vida pessoal e não apenas a dimensão física, mas também a emocional e social, haja vista tratar-se de uma patologia que está associada à alta morbidade, mortalidade e cronicidade (Thumboo \& Strand, 2007). Com prognóstico incerto, pode

1 Endereço para correspondência: Av. Tancredo Neves n 909 , Ed. André Guimarães Business Center, sala 1203. Caminho das Árvores, Salvador, BA. CEP: 41820.021. Fone: (71) 3341.2320.Email: silviacal@uol. com.br levar à incapacitação, além de ser potencial causa de desfiguração (Pájaro, 2005). O estresse emocional decorrente dessa condição e o uso de corticóides utilizados no tratamento do LES têm sido responsabilizados por boa parte de suas manifestações psicopatológicas (Miguel et al., 1994; Nery et al., 2007).

O fator etiológico das alterações psicopatológicas no LES ainda não está suficientemente esclarecido, apesar do aprimoramento das pesquisas nessa área, mas há consenso entre a comunidade científica com relação à etiologia da doença, como sendo de origem multifatorial, ou seja, fatores hormonais (estrogênio), genéticos, ambientais (radiação ultravioleta, medicamentos), infecciosos (virais) e estresse psicológico. Pesquisadores concluíram que os fatores psicológicos, incluindo traços de personalidade, são codeterminantes, desencadeantes, exacerbadores ou patoplásticos do LES (Ayache \& Costa, 2005).

A comorbidade psiquiátrica não tratada está associada à morbidade, dificuldade de adesão ao tratamento e aumento da utilização dos serviços médicos. Muitos desses casos têm tratamento efetivo, e em relação à depressão, quando tratada adequadamente, pode diminuir a taxa de morbi-mortalidade em 70\% (Fleck, 2003). Há ainda muitos casos de suicídio entre os pacientes com LES, que possuem cinco vezes mais chances de cometer suicídio quando comparados à população geral (Karassa, Magliano \& Isenberg, 2003). 
Matsukawa, Sawada e Issenberg (1994) estudaram sete pacientes com LES que cometeram suicídio e observaram que todos os pacientes cometeram o ato próximo ao período da admissão (média de 20 dias), sendo a maior parte em uso de esteróides, sugerindo que a atividade da doença não estava totalmente controlada quando do momento do suicídio. Não havia, no entanto, sinais claros de iminente suicídio. Por fim, os autores recomendaram que os pacientes fossem acompanhados (após a admissão) por, no mínimo, dois meses para prevenir suicídio.

Entre os fatores que poderiam levar uma pessoa doente fisicamente à psicopatologia estão o agravamento de conflitos intrapsíquicos, a inadequação dos mecanismos de defesa, a diminuição da auto-estima, a alteração da imagem corporal, a ruptura do ciclo sono-vigília, o uso de medicamentos e de procedimentos que afetam o sistema nervoso central (Botega \& Smaira, 2006). Por outro lado, eventos de vida estressantes também podem influenciar o início de doenças e a imunossupressão, acentuando a vulnerabilidade do indivíduo.

A enfermidade não é somente uma mudança física, mas uma quebra no curso normal da vida que modifica o ritmo e a direção pessoal da existência (Araújo, 2004), o que, muitas vezes, corresponde a uma condição que retira o indivíduo de um lugar estável, desestruturando seu modo de vida, refletindo ameaças que podem chegar a vivências catastróficas, uma vez que representam interrupções de planos, afastamento da família e trabalho e, consequentemente, mobilizando sentimentos como ansiedade, tristeza, medo e angústia (Figueiredo, Giglio \& Botega, 2006). Essa condição consiste, muitas vezes, numa ruptura que conduz a pessoa a buscar um novo sentido para sua situação, a rever todos os seus valores anteriores e definir prioridades em função desse momento, de suas limitações e possibilidades. Nesse sentido, a crise pode ser vivenciada como uma oportunidade de descobrir seu potencial de superação, aproveitando a ressignificação que se processa a partir da nova realidade e de suas limitações, mas também como oportunidade de reconhecer-se como alguém responsável pelo desenvolvimento de sua enfermidade, ou seja, identificando sua implicação em todo esse processo.

Considerando-se que a experiência de adoecer é algo muito subjetivo e dinâmico e que ela pode apresentar diversas formas de expressão, acredita-se que o sentido atribuído a este processo poderá interferir no tratamento e na forma de vivenciar esse momento de crise. Subjetividade diz respeito ao sistema de significações e ao sentido subjetivo com os quais se organiza a vida psíquica do indivíduo e tal conceito não se esgota no campo intrapsíquico individual, mas inclui também os processos sociais (Rey, 2005).

Tanto na neurose como na psicose os sintomas de natureza somática ou psíquica são originados nos complexos. Complexo é uma coleção de várias ideias mantidas juntas por um tom emocional comum a todas. Assim, quando um complexo se constela, não há apenas alteração fisiológica, mas em toda a estrutura corporal, alteração esta que pode não ser percebida de imediato pela pessoa, sendo que muitas vezes se apresenta como um mal-estar ou através de uma sintomatologia mais definida (Ramos, 1990).
A auto-imagem corporal, assim como todas as sensações sinestésicas presentes na consciência fazem parte do complexo, e para sua formação entram contribuições anatômicas, fisiológicas, neurológicas e sociológicas (Capisano, 1992). Torna-se importante compreender os fenômenos em nível físico, buscando-se não só a causa dos sintomas, mas o processo de geração de sentido (Araújo, 2004).

Há uma distinção entre sentido e significado; significado corresponde ao sistema de relações semânticas em que a construção ocorre no processo histórico, enquanto que o sentido corresponde aos aspectos construídos pelo indivíduo através de seu processo de socialização. Portanto, somente a pessoa, através do processo de significação próprio de seu contexto, pode definir o sentido que a doença tem para si e quais as implicações que acarreta em sua vida. Ainda que a doença seja a mesma, cada pessoa constrói sentidos diferentes, e estes sentidos são dinâmicos, podendo mudar a depender da peculiaridade da doença e da rede de significações que fazem parte de seu cotidiano (médicos, outros pacientes com LES, amigos, vizinhos, familiares etc (Araújo \& Azucena, 2007).

$\mathrm{O}$ entendimento da relação da pessoa com sua própria doença constitui uma contribuição importante da Psicologia na interface com a saúde, pois permite identificar recursos no campo psicossocial capazes de auxiliar o indivíduo doente a lidar melhor com esse sofrimento (Araújo \& Azucena, 2007).

O sintoma corporal pode ser visto como um símbolo que expressa a relação psique-corpo. Compreender o significado desse símbolo inclui a busca da causa e de sua finalidade, numa amplificação do significado que levará a uma maior integração de conteúdos inconscientes no ego (Ramos, 1994), tarefa esta que exige um profissional especializado. A necessidade de intervenção psicoterapêutica nas diversas unidades de assistência médica tem sido cada vez mais reconhecida e aceita no campo da medicina psicossomática (Fritzche, Struss, Stein \& Spain, 2003).

As psicoterapias correspondem a um universo amplo de práticas discursivas, baseadas na comunicação e no relacionamento sistematizado com finalidade terapêutica. Tal amplitude varia desde o apoio psicológico, numa escuta acolhedora e de empatia com o sofrimento do paciente, a uma maior investigação do seu mundo subjetivo. A abordagem psicoterápica feita num hospital não deve ser confundida com a psicoterapia realizada por especialista num consultório, pois principalmente nessa situação, apoio é fundamental, sendo necessárias técnicas mais rápidas, mais diretivas, de curta duração com metas mais definidas e estratégias específicas, respeitando-se mais do que nunca as defesas do paciente, devido à fragilização decorrente da doença (Figueiredo et al., 2006).

A intervenção psicoterapêutica do paciente em crise, principalmente do paciente hospitalizado, deve ter como objetivo auxiliá-lo a retornar o funcionamento anterior à crise e encontrar formas adaptativas para a nova situação, ou seja, enfocar o aqui e agora que corresponde à psicoterapia de apoio, mas devem ser consideradas as limitações relacionadas ao paciente, cuja personalidade deve ser bem estruturada, apresentar capacidade de abstração e de estabelecer aliança terapêutica, além de ter motivação para a psicoterapia. 
Nesse sentido, há que se considerar também as limitações do terapeuta relativas a sua formação, vivência profissional, disponibilidade e capacidade para suportar angústia, e empatizar com determinados pacientes (Figueiredo et al., 2006).

Para a Organização Mundial de Saúde, a existência do sofrimento psíquico ou da doença mental e a motivação para a psicoterapia são os aspectos mais importantes e determinantes da necessidade da intervenção psicológica, podendo interferir no desempenho da mesma (Spahn et al., 2002).

$\mathrm{O}$ envolvimento dos aspectos psicológicos no adoecer do paciente com lúpus foi evidenciado por diversas pesquisas (Ayache \& Costa, 2005; Nery et al., 2008; Cal, Borges \& Santiago, 2006), encontrando-se uma prevalência de depressão em $62 \%$ dos pacientes de ambulatório de Reumatologia (Cal et al., 2006), utilizando-se como instrumento o PRIME-MD (Spitzer, Kroenke \& Williams, 1999).

A depressão, muitas vezes, não é diagnosticada pelos médicos pelo fato deles acharem que os sintomas depressivos são uma resposta natural à doença física, capaz de ameaçar a vida de alguém, ou, numa posição oposta, é dado o diagnóstico de depressão a pacientes com tristeza ou com sintomas físicos causados pela doença de base (Botega, Furlanetto \& Fráguas Jr., 2006). Para evitar esse tipo de confusão, Cavanaugh (conforme citado por Von Ammon, 1995) sugeriu observar que sintomas como fadiga e alterações do sono, do apetite, do peso e da psicomotricidade ajudam a corroborar o diagnóstico da depressão quando são excessivos em relação ao esperado para sua doença ou tratamento, ou quando surgem associados aos sintomas cognitivos e afetivos da depressão (Von Ammon, 1995). Ansiedade e outras síndromes psiquiátricas são também comuns no LES (Nery et al., 2008).

$\mathrm{O}$ apoio psicológico foi citado, embora de forma superficial, entre as medidas gerais recomendadas por artigo científico de diretriz, elaborado por equipe conceituada composta de oito reumatologistas, um nefrologista e um dermatologista que trabalham em grandes centros de atendimento ao LES, vários deles com publicações científicas na área (Sato et al., 2006).

Apesar do reconhecimento da importância do acompanhamento psicológico do paciente com LES, relatos de intervenção nessa área são escassos. O principal objetivo deste trabalho foi realizar revisão de literatura sobre a eficácia da intervenção psicológica no tratamento do paciente com LES, além de proporcionar uma reflexão sobre o papel do psicólogo no ambulatório de Reumatologia.

\section{Método}

Trata-se de uma revisão sistemática, através de pesquisa nas bases de dados Pubmed, Biblioteca Virtual de Saúde (BVS), Pepsic (Periódicos eletrônicos em psicologia), LIS-Psi (Localizador de informação em saúde), em que foram buscados estudos publicados até junho de 2009, utilizando-se os descritores "psychotherapy" and "lupus". Devido ao pequeno número de artigos publicados, foram incluídos todos os principais artigos (ensaios clínicos randomizados e estudos prospectivos) encontrados, num total de seis, sendo quatro ensaios clínicos randomizados e dois estudos prospectivos. Foram excluídos os artigos de revisão e os estudos de casos.

\section{Resultados e Discussão}

A Tabela 1 apresenta os artigos que foram incluídos nesta revisão. Mais especificamente, a tabela apresenta os autores, o tipo de intervenção realizada, o desenho do estudo e os resultados alcançados.

As abordagens citadas foram divididas em intervenções psicoeducacionais e acompanhamento psicoterápico, sendo que as psicoeducacionais foram relativas a programas assistenciais focados em fornecer informação sobre os sintomas e as abordagens terapêuticas, com maior ênfase nas respostas de coping definidas como o conjunto de estratégias utilizadas pelas pessoas para se adaptarem a circunstâncias adversas ou estressantes (Antoniazzi, Dell'Aglio, \& Bandeira, 1998), no manejo diário do paciente no curso da doença, e foram realizadas por profissionais diversos, como médicos e enfermeiros. As abordagens psicoterápicas incluíram apoio por telefone $\mathrm{e}$ psicoterapias breves, sendo que um dos estudos utilizou as duas abordagens simultaneamente (Haupt et al., 2005).

As intervenções psicoterápicas foram, na sua maioria, em grupo e por um tempo reduzido, de seis sessões a nove meses, o que pode levar, em alguns casos, a uma abordagem mais superficial das dificuldades emocionais do paciente. Se, por um lado a proposta grupal oferece a oportunidade de reconhecimento do caráter comum ao sofrimento do paciente, funcionando, muitas vezes, como um espelho, pois os pacientes têm a oportunidade de se verem refletidos em suas semelhanças, e de se solidarizar em torno de uma problemática comum, por outro lado pode inibir pelo fato de falarem de coisas mais íntimas e pessoais. Alguns autores indicam um acompanhamento individual para estes pacientes, uma vez que vários deles têm sérios problemas psicológicos, o que do ponto de vista funcional torna-se difícil em se tratando de ambulatórios com um grande número de pacientes (Nowicka-Sauer, 2007; Geiger \& Langewitz, 2007).

Apesar de os resultados serem positivos, em sua maioria, em relação à melhora na habilidade de coping e da qualidade de vida (Haupt et al., 2005) e curso da doença menos agressivo (Edworthy et al., 2003), não houve uma avaliação da associação entre esses resultados e a atividade da doença e nem com os medicamentos que estavam em uso no período do acompanhamento psicológico, o que pode implicar na ocorrência de vieses. Novas pesquisas serão importantes para avaliar estes aspectos.

A escassez de pesquisas e publicações que contemplem uma visão psicodinâmica do LES pode ser reflexo da ausência de política mais ampla quanto à inclusão do psicólogo nos ambulatórios de Reumatologia e de programas de assistência psicológica voltados para essa população, e mais ainda no que se refere à consciência dos benefícios desse tipo de abordagem entre os médicos e os próprios pacientes.

O acompanhamento psicológico, independente da técnica utilizada ou de sua fundamentação teórica, pode trazer benefícios para o paciente no sentido de maior adaptação ao LES, redução da dor e agravamento da doença, habilidades de coping, melhora da depressão e ansiedade, além de mudanças 
Tabela 1. Ensaios clínicos e estudos prospectivos que avaliaram o efeito da intervenção psicológica em pacientes com LES.

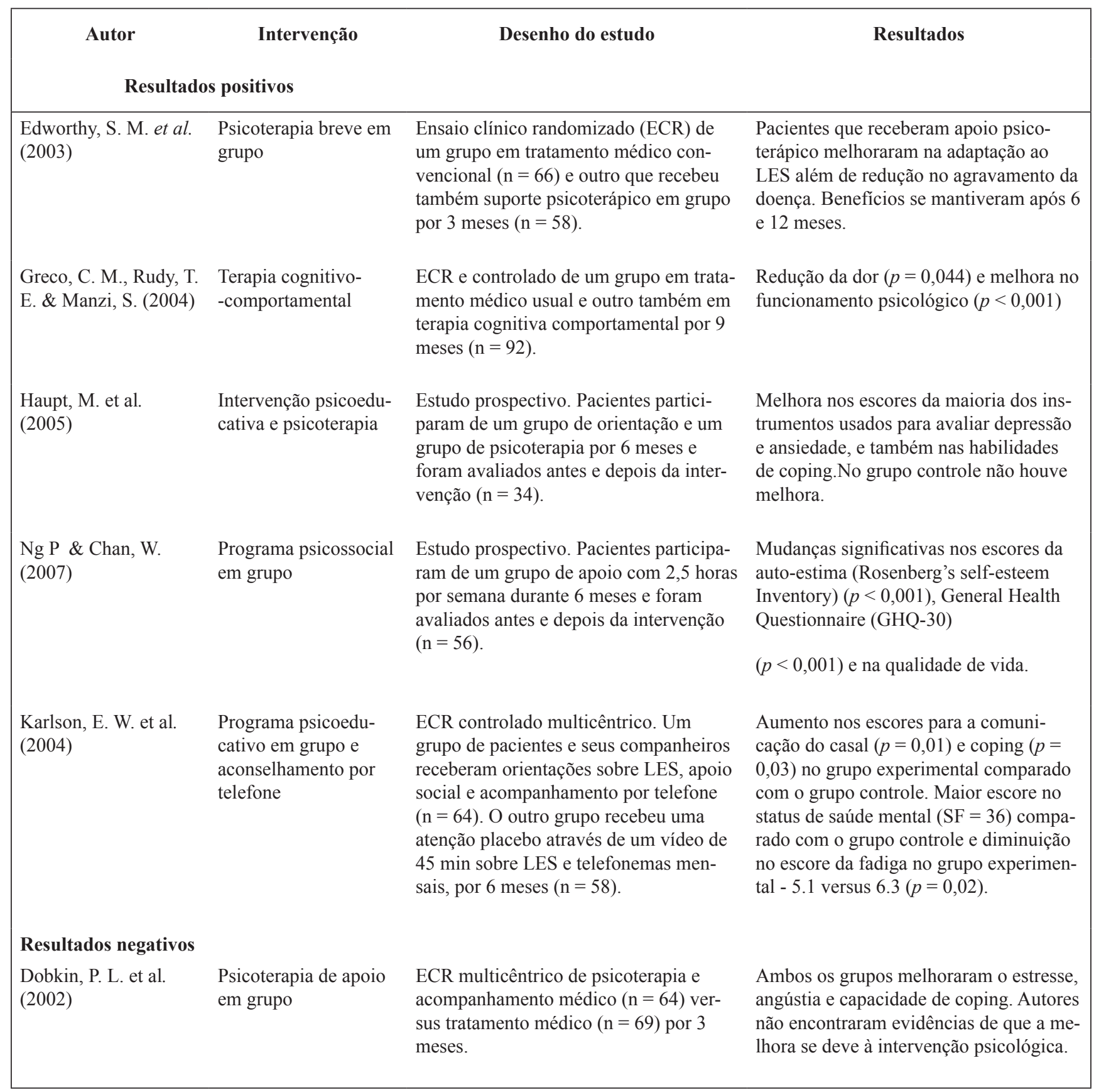

significativas nos escores da auto-estima e na qualidade de vida do paciente e, portanto, pode ser uma ferramenta importante, capaz de auxiliar de forma significativa o trabalho da equipe médica, o que reforça a importância da equipe multiprofissional atuando num ambulatório de Reumatologia.

\section{Referências}

Abdel-Nasser, A. M., Ghaleb, R. M., Mahmoud, A., Khairy, W., \& Mahmoud, R. M. (2008). Association of anti-ribosomal $\mathrm{P}$ protein antibodies with neuropsychiatric and other manifestations of systemic lupus erythematosus. Clinical Rheumatology, 27, 1377-1385.
Ampelas, J. F., Wattiaux, M. J., \& Van Amerongen, A. P. (2001). Psychiatric manifestations of lupus erythematosus systemic and Sjogren's syndrome. L'Encephale 27, 588-599.

Antoniazzi A. S, Dell' Aglio D. D, \& Bandeira D. R. (1998). O conceito de coping: uma revisão teórica, Estudos de Psicologia (Natal), 3, 273-294.

Araújo, A. D. (2004). A doença como ponto de mutação: os processos de significação em mulheres portadoras de lúpus eritematoso sistêmico. Dissertação de Mestrado, Universidade Federal do Rio Grande do Norte, Natal.

Araújo, A. D., \& Azucena, M. (2007). Expressões e sentidos do lúpus eritematoso sistêmico (LES). Estudos de Psicologia (Natal), 12, 119-127. 
Ayache, D., \& Costa, I. P. (2005). Alterações da personalidade no lúpus eritematoso sistêmico. Revista Brasileira de Reumatologia, 45, 313-8.

Botega, N., Furlanetto, L., \& Fráguas Jr., R. (2006). Depressão. In N. J. Botega (Ed.), Prática psiquiátrica no hospital geral: interconsulta e emergência (2 ${ }^{\mathrm{a}}$ ed.), (pp. 225-246). Porto Alegre: Artmed.

Botega, N., \& Smaira, S. (2006). Morbidade psiquiátrica no hospital geral. In N. J. Botega (Ed.), Prática psiquiátrica no hospital geral: interconsulta e emergência ( $2^{\mathrm{a}}$ ed.), (pp. 35-47). Porto Alegre: Artmed.

Cal, S. F., Borges, A. P., \& Santiago, M. B. (2006). Prevalência e classificação da depressão em pacientes com lúpus eritematoso sistêmico atendidos em um serviço de referência da cidade de Salvador. Jornal da LIRRNE, 2, 36-42.

Capisano, H. (1992). Imagem corporal. Em J. Mello Filho (Org.), Psicossomática Hoje (pp. 179-192). Porto Alegre: Artmed.

Dobkin, P. L., Da, C. D., Joseph, L., Fortin, P. R., Edworthy, S., Barr, S., Esdaile, J. M., Beaulieu, A., Zummer, M., Senécal, J. L., Goulet, J. R., Choquette, D., Rich, E., Smith, D., Cividino, A., Gladman, D., St-Pierre, Y., \& Clarke, A. E. (2002). Counterbalancing patient demands with evidence: results from a pan-Canadian randomized clinical trial of brief supportiveexpressive group psychotherapy for women with systemic lupus erythematosus. Annals Behavioral Medicine, 24, 88-99.

Eber, T., Chapman, J., \& Shoenfeld, Y. (2005). Anti-ribosomal P-protein and its role in psychiatric manifestations of systemic lupus erythematosus: myth or reality? Lupus, 14, 571-575.

Edworthy, S. M., Dobkin, P. L., Clarke, A. E., Da, C. D., Dritsa, M., Fortin, P. R.Barr, S., Emsworth, J. M., Beaulieu M., Z., Senecal, J., Goulet, J., Choquette, D., Rich, E., Smith, D., Cividino, A., Gladman, D., \& Devins, G. M. (2003). Group psychotherapy reduces illness intrusiveness in systemic lupus erythematosus. Journal of Rheumatology, 30, 1011-1016.

Figueiredo, J., Giglio, J., \& Botega, N. (2006). Tratamento psicológico: psicoterapia de apoio, relaxamento, meditação. In N. J. Botega (Ed.), Prática psiquiátrica no hospital geral: interconsulta e emergência ( $2^{\mathrm{a}}$ ed.), (pp. 484-496). Porto Alegre: Artmed.

Fleck, M. A. (2003). Diretrizes da Associação Médica Brasileira para o tratamento da depressão. Revista Brasileira de Psiquiatria, 25, 114-122.

Fritzche, K., Struss, Y., Stein B., \& Spain, C. (2003). Psychosomatic liaision serve in hematological oncology: need for psychotherapeutic intervention and their realization. Hematological Oncology, 21, 83-89.

Geiger, I., \& Langewitz, W. (2007). Psychotherapy in a patient with lupus erythematodes - a disease with an uncertain course. Therapeutische Umscha, 64, 581-584.

Greco, C. M., Rudy, T. E., \& Manzi, S. (2004). Effects of a stress-reduction program on psychological function, pain, and physical function of systemic lupus erythematosus: a randomized controlled trial. Arthritis \& Rheumatism, 51, 625-634

Haupt, M., Millen, S., Janner, M., Falagan, D., Fischer-Betz, R., \& Schneider, M. (2005). Improvement of coping abilities in patients with systemic lupus erythematosus: a prospective study. Annals of the Rheumatic Diseases, 64, 1618-1623.
Karassa, F. B., Magliano, M., \& Isenberg, D. A. (2003). Suicide attempts in patients with systemic lúpus erythematosus. Annals of the Rheumatic Diseases, 62, 58-60.

Karlson, E. W., Liang, M. H., Huang, J., Fitzgerald, I., Rogers, M. P., \& Daltroy, L. H. (2004). A randomized clinical trial of a psychoeducational intervention to improve outcomes in systemic lupus erythematosus. Arthritis \& Rheumatism, 50, 1832-1841.

Lessa, B., Santana, A., Lima, I., Almeida, J. M., \& Santiago, M. B.(2006). Prevalence and classification of headache in patients with systemic lupus erythematosus. Clinical Rheumatology, $25,850-853$.

Matsukawa Y., Sawada S., \& Issenberg D. A. (1994). Suicide in patients with systemic lúpus erythematosus: a clinical analysis of seven suicidal patients. Lupus, 3, 31-35.

Miguel, E. C., Pereira, R. M., Pereira, C. A., Baer, L., Gomes, R. E., de Sa, L. C., Hirsch, R., Barros, N. G., Navarro, J. M., \& Gentil, V. (1994). Psychiatric manifestations of systemic lupus erythematosus: clinical features, symptoms, and signs of central nervous system activity in 43 patients. Medicine, 73, 224-232.

Nery, F. G., Borba, E. F., Hatch, J. P., Soares, J. C., Bonfa, E., \& Neto, F. L. (2007). Major depressive disorder and disease activity in systemic lupus erythematosus. Comprehensive Psychiatry, 48 (1), 14-19.

Nery, F. G., Borba, E. F., Viana, V. S., Hatch, J. P., Soares, J. C., Bonfa, E., \& Neto, F. L. (2008). Prevalence of depressive and anxiety disorders in systemic lupus erythematosus and their association with anti-ribosomal $\mathrm{P}$ antibodies. Progress in Neuro-Psychopharmacology \& Biological Psychiatry Journal, 32, 695-700.

Ng, P., \& Chan, W. (2007). Group psychosocial program for enhancing psychological well-being of people with systemic lupus erythematosus. Journal of Social Work in Disability \& Rehabilitation, 6, 75-87.

Nowicka-Sauer, K. (2007). Patients' perspective: lupus in patients' drawings. Assessing drawing as a diagnostic and therapeutic method. Clinical Rheumatology, 26, 1523-1525.

Olsen, M. L., O'Connor, S., Arnett, F. C., Rosenbaum, D., Grotta, J. C., \& Warner, N. B. (1991). Autoantibodies and rheumatic disorders in a neurology inpatient population: a prospective study. American Journal of Medicine, 90, 479-488.

Pájaro, S. (2005). Lupus eritematoso sistémico: síndrome de Cushing y Psiquiatría de enlace. Revista Facultad de Medicina (Bogotá), 53, 117-123.

Ramos, D. (1990). A psique do coração: uma leitura analítica do seu simbolismo (10 a ed.). São Paulo: Editora Cultrix Ltda.

Ramos, D. (1994). A psique do corpo: uma compreensão simbólica da doença ( $2^{\mathrm{a}}$ ed.). São Paulo: Summus editorial Ltda.

Rey, G. F. (2005). Pesquisa Qualitativa em psicologia, caminhos e desafios. São Paulo: Pioneira Thompson Learning Ltda.

Sato, E. I., Bonfá, E. D., Costallat, L. T., Silva, N. A., Brenol, J. C., Santiago, M. B., Szajubok, J. C., Rachid-Filho, A., Barros, R. T., \& Vasconcelos, M. (2006). Lúpus eritematoso sistêmico: acometimento cutâneo/articular. Revista da Associação Médica Brasileira, 52, 384-386.

Spahn, C., Beker, B., Fritzsche K., Burger T., Blum, H. E., \& Wirsching, M, (2002). Psychosomatic liaison service in Medicine - need for psychotherapeutic interventions and then realization. Swiss Medical Weekly, 133, 166-173. 
Spitzer, R. L., Kroenke, K., \& Williams, J. B. (1999). Validation and utility of a self-report version of PRIME-MD: the PHQ primary care study. Primary Care Evaluation of Mental Disorders. Patient Health Questionnaire. JAMA, 282, 1737-1744.

Thumboo, J., \& Strand, V. (2007). Health-related quality of life in patients with systemic lupus erythematosus: an update. ANNALS Academy of Medicine Singapore, 36, 115-122.

Von Ammon, C. S. (1995). Depression in the medically ill. Critical issues in diagnostic assessment. Psychosomatics, 36, 48-59.

\section{Conferencia Internacional de Psicología de la Salud}

18 de Novembro de 2011

Havana, CU 